Revista electrónica publicada por el Centro Centroamericano de Población, Universidad de Costa Rica, 2060 San José, Costa Rica http://ccp.ucr.ac.cr

Población y Salud en Mesoamérica Revista electrónica semestral, ISSN-1659-0201

Volumen 10, número 2, informe técnico 1

Enero - junio, 2013

Publicado 1 de enero, 2013

http://ccp.ucr.ac.cr/revista/

\title{
A propósito del SIDA: un estudio cualitativo sobre percepción $y$ actitudes de estudiantes $y$ profesorado universitario en Chontales, Nicaragua
}

\author{
David Cobos-Sanchiz \\ Juan Agustín Morón-Marchena \\ Emilio José López-Jarquín \\ Encarnación María Reyes-Costales
}

\section{(cc) $\mathrm{EY}-\mathrm{HE}-\mathrm{HD}$}

Protegido bajo licencia Creative Commons

Centro Centroamericano de Población 


\title{
A propósito del SIDA: un estudio cualitativo sobre percepción y actitudes de estudiantes y profesorado universitario en Chontales, Nicaragua
}

\section{On the subject of AIDS: a study on qualitative perceptions and attitudes of college students and lecturers in Chontales, Nicaragua

\author{
David Cobos-Sanchiz ${ }^{1}$ Juan Agustín Morón-Marchena ${ }^{2}$,Emilio José López-Jarquín ${ }^{3}$, \\ Encarnación María Reyes-Costales ${ }^{4}$
}

\section{RESUMEN}

El objetivo principal de este artículo es profundizar en las percepciones y actitudes sobre el VIH de la comunidad universitaria nicaragüense. Se trata de un estudio cualitativo desarrollado en la Universidad Nacional Autónoma de Nicaragua-Managua, a partir de varios grupos de discusión. Para el análisis de los datos se ha empleado la técnica de análisis de contenido y el programa de análisis cualitativo Atlas.ti, obteniendo como resultado una red conceptual de códigos relacionados. Entre los hallazgos más significativos se destaca el bajo nivel de conocimientos sobre ITS que sigue habiendo entre profesorado y estudiantes, así como la persistencia de numerosos mitos en relación a las personas con VIH, lo que provoca que la discriminación social siga siendo un tema de interés comunitario. El conjunto de conclusiones del estudio sirve para informar y orientar futuras actividades de Educación y Promoción de la Salud en el contexto en el que se desarrolla la investigación.

Palabras clave: VIH; Educación Sanitaria; Percepción Social; Estigma Social.

\begin{abstract}
The main objective of this paper is to analyze the perceptions and attitudes about the HIV of the Nicaraguan university community. This is a qualitative study developed at the National Autonomous University of Nicaragua, Managua, from various discussion groups. To analyze the data, the content analysis technique and the qualitative analysis programme Atlas.ti have been employed, resulting in a conceptual network of related codes. Among the most significant findings, the low level of knowledge about STI among teachers and students can be highlighted, as well as the persistence of numerous myths regarding people with HIV, causing that social discrimination is still a topic of interest for the community. The overall conclusions of the study are useful to inform and guide future activities of Education and Health Promotion in the context in which research is conducted
\end{abstract}

Keywords: HIV; Health Education; Social Perception; Social Stigma.

Recibido: 28 set. 2012

Aprobado: 22 oct. 2012

\footnotetext{
${ }^{1}$ Universidad Pablo de Olavide, de Sevilla. Facultad de Ciencias Sociales. ESPAÑA. dcobos@upo.es

${ }^{2}$ Universidad Pablo de Olavide, de Sevilla. ESPAÑA. jamormar1@upo.es

${ }^{3}$ Universidad Nacional Autónoma de Nicaragua. NICARAGUA. ejlopezj@unan.edu.ni

${ }^{4}$ Bärkraft Assistans AB. SUECIA. encreycos@gmail.com
} 


\section{INTRODUCCION}

Desde el inicio de la epidemia, más de sesenta millones de personas se han infectado y casi treinta millones han fallecido por causas relacionadas con el VIH (UNAIDS, 2010), lo que ha supuesto un problema mundial de tremendo calado desde cualquier punto de vista desde el que se enfrente: de salud pública y laboral, socio-económico y, en definitiva, como un auténtico lastre para el desarrollo de los pueblos. A pesar de todo hay motivos para la esperanza, puesto que el esfuerzo empleado durante las últimas décadas ha supuesto que, por primera vez, las tendencias sobre contagios y fallecimientos se empiecen a invertir. Al menos 56 países se han estabilizado $\mathrm{u}$ obtenido descensos significativos en las tasas de nuevas infecciones por VIH en los últimos años. El último informe del ONUSIDA (UNAIDS, 2010) indica que se ha conseguido detener la epidemia de VIH y que, a nivel general, se comienza a reducir la propagación del virus. Las nuevas infecciones han caído en torno a un $20 \%$ en la última década, las muertes relacionadas se han reducido alrededor de un $20 \%$ en los últimos cinco años y el número total de personas con VIH se está estabilizando. Así mismo, las personas con VIH viven más tiempo y las muertes relacionadas con el virus descienden gracias a la ampliación del tratamiento.

Sin embargo, no conviene bajar la guardia. La reciente estabilización de la epidemia mundial no puede ni debe ocultar su aspecto más dramático: la enorme cifra de víctimas que se sigue cobrando. Además, hay que tener en cuenta que la historia de las enfermedades infecciosas sugiere que las epidemias muchas veces son cíclicas, caracterizadas por oleadas de infección que dificultan la predicción del futuro curso de la epidemia. De hecho, la propia epidemia de VIH ha desafiado reiteradamente las predicciones derivadas de modelizaciones epidemiológicas (May y Anderson, 1979). Siguiendo el informe precitado, los datos absolutos actuales para América Central y del Sur son estos: actualmente 1,4 millones de personas viven con VIH (WHO, 2010). Se observa una disminución importante de nuevas infecciones entre niños menores de quince años y hay un aumento considerable en la aplicación de medicamentos antirretrovíricos en mujeres embarazadas que viven con VIH para prevenir la transmisión a su descendencia. En cambio, las relaciones sexuales entre hombres siguen siendo una causa clave en la transmisión del VIH. La mayoría de epidemias de VIH en América Central y del Sur se concentran en redes de hombres que mantienen relaciones sexuales con hombres y alrededor de estas redes.

En el caso concreto de Nicaragua, se ha modificado en las últimas décadas su perfil demográfico y experimentado una transición epidemiológica, donde la epidemia del VIH es un contribuyente de este perfil. Desde 1987 a 2009, el comportamiento de la epidemia ha evolucionado a una epidemia concentrada, la cual se ha mantenido en esta clasificación similar a lo reportado en el informe para ONUSIDA sobre el seguimiento de progresos en los países (UNGASS) (Gobierno de Nicaragua, 2010). La tasa de incidencia nacional para 2009, fue superada en algunos Departamentos que presentan mayores riesgos de infección por VIH, entre ellos Chinandega, Managua y Masaya. En definitiva, aunque el país sigue teniendo, como acabamos de decir, una epidemia concentrada, hay Departamentos y municipios que comparten características sociodemográficas y económicas que están notificando un incremento del número de casos relacionados con el fenómeno migratorio, por la existencia de puestos de pasos y sitios de atractivo turístico. 
La principal vía de transmisión -siguiendo las mismas pautas generales que hemos descrito anteriormente para el conjunto de América Central y del Sur- continúa siendo la sexual con el $86 \%$. Se identifica una evolución positiva en la respuesta nacional, considerando que el Ministerio de Salud ha dirigido todos sus esfuerzos en mejorar la calidad y la atención en salud, con la transformación e implementación del nuevo Modelo de Atención en Salud Familiar y Comunitario (MOSAFC) y la transversalización de las intervenciones en salud, con un enfoque de respuestas multisectoriales ante el VIH. Según las mismas fuentes gubernativas, en el comportamiento de la epidemia en Nicaragua se identifican dos poblaciones claves que requieren intervenciones adecuadas para lograr cambios importantes: la población más expuesta debido a prácticas de mayor riesgo (hombres que tienen sexo con hombres, trabajadoras sexuales, uniformados, poblaciones móviles, privados de libertad y usuarios de drogas intravenosas) y la población vulnerable a la epidemia debido a factores externos a su control (niños, niñas, adolescentes y jóvenes).

En este contexto se inserta la investigación que se recoge en este artículo y, en concreto, sobre el segundo segmento poblacional descrito (jóvenes y adolescentes). Diversas investigaciones han puesto de manifiesto que la mayoría de adolescentes y jóvenes presentan déficits en el nivel de autoeficacia y susceptibilidad frente al VIH lo que, sumado a las características propias de su edad, desencadenan comportamientos de riesgo que los hacen especialmente vulnerables a la infección por VIH (Uribe, Vergara y Barona, 2009). También se ha referido que la percepción del riesgo en estudiantes universitarios no coincide con la conducta sexual y la protección ante el VIH, debido a que continúan teniendo relaciones sexuales sin protección, a pesar de percibir el riesgo de adquirir esta infección (Idem).

Este aspecto es de especial relevancia puesto que en las estrategias de prevención, tradicionalmente se ha partido de la base de que existe una alta correlación entre la percepción del riesgo y el comportamiento. Por tanto, una primera estrategia (muy lógica, por otra parte) es la de desarrollar campañas informativas extensas en la idea de que conocer los riesgos es el mejor camino para después poder prevenirlos. Por supuesto, esto es fundamental porque no se puede prevenir lo que no se conoce pero las variables que explican los comportamientos humanos son mucho más complejas y tienen que ver con la construcción social de los riesgos. Al igual que no existe un modo universalmente compartido de interpretar la realidad social, la percepción del riesgo implica creencias, juicios y sentimientos, así como valores y la disposición en sentido amplio que las personas adoptan frente a los riesgos y sus beneficios o perjuicios. La percepción del riesgo es multidimensional: un riesgo concreto significa cosas distintas para personas distintas y cosas distintas en contextos diferentes. La percepción del riesgo, por tanto, es un fenómeno de carácter humano y social que tiende a estar influenciada por la cultura, los argumentos políticos o los económicos tanto como los científicos (Rodríguez, Ballesteros, Mejías y Rodríguez-Felipe, 2008).

Las creencias y valores compartidos por los grupos sociales influyen en lo que se considera o no como riesgo, aspecto de vital importancia en la adolescencia y juventud. En cierto modo se podría decir que cada grupo social selecciona los riesgos que quiere prevenir y los que está dispuesto a asumir. En ocasiones se llega a sobredimensionar un riesgo para la salud o se minimiza otro, sin tener en cuenta en absoluto las evidencias científicas (Idem). Para ejemplificar esta idea podemos traer a colación un estudio desarrollado por un grupo de investigadoras de la Universidad de Granada que pone de manifiesto que, a pesar de todos los esfuerzos informativos 
y formativos desarrollados en las últimas décadas, el riesgo de infección por VIH ha aumentado entre las mujeres españolas en los últimos años por la disminución de la percepción del riesgo, lo que ha conllevado un relajamiento en la adopción de medidas preventivas (Teva, Bermúdez y Buela, 2009).

En este sentido, para la prevención del VIH entre adolescentes y jóvenes, consideramos que el trabajo de promoción de la salud sobre la percepción del riesgo de estos colectivos es un elemento fundamental que ha de hacer hincapié en la autoeficacia percibida, ya que ésta permite implantar un comportamiento deseado y generar la motivación que se requiere para lograr y mantenerlo (Noboa y Serrano-García, 2006). Nuestro grupo de investigación viene trabajado bajo estas premisas a través de diversas iniciativas en el ámbito de la Educación, la Promoción de la Salud y la Educación para el Desarrollo, disciplinas donde hemos volcado un buen número de esfuerzos en los últimos años. De hecho, este trabajo es sólo una muestra más de la estrecha colaboración que, con carácter continuado, la Universidad Pablo de Olavide, de Sevilla (España) viene desarrollando con la UNAN-Managua (Nicaragua) desde hace ya más de un quinquenio y que se pone de manifiesto en la realización e implementación de numerosos proyectos de Investigación, Formación y Cooperación Universitaria al Desarrollo, especialmente con la Facultad Autónoma Regional Multidisciplinar (FAREM) de Chontales que se describen profusamente en otros trabajos anteriores (López, Cobos y Morón, 2011; Morón, López y Cobos, 2011).

\section{MATERIAL Y MÉTODOS}

El estudio es continuación y profundización de una investigación sobre actitudes, percepciones y prácticas de riesgos de estudiantes y profesorado de la FAREM-Chontales de la UNANManagua, cuyos primeros análisis ya se recogen ampliamente en otra publicación anterior (Morón, López y Cobos, 2011) y que tenía como principal objetivo "generar evidencia científica de la situación de las poblaciones de estudiantes y profesorado de la FAREM-Chontales con relación a VIH, que permita informar intervenciones dirigidas a mejorarlas". Como quiera que los estudios iniciales eran meramente cuantitativos, nos pareció necesario profundizar mucho más en el análisis cualitativo de las percepciones y actitudes de estudiantado y profesorado, al objeto de conseguir la triangulación metodológica que asegurara la validez técnica formal de las conclusiones obtenidas, así como lograr una mayor riqueza en los resultados. Para ello desarrollamos tres grupos de discusión que se celebraron entre el 12 y el 19 de febrero de 2010 . Mediante esta técnica se persigue analizar las ideas de cada colectivo de personas mediante el contraste de pareceres entre ellas.

Concretamente, pretendíamos acercarnos a entender cómo viven profesores y estudiantes esta problemática desde su propia subjetividad. Los grupos de discusión nos ofrecen la posibilidad de analizar los discursos construidos de forma subjetiva pero sobre unas pautas sociales, es decir, captan las representaciones y los valores compartidos por ambos colectivos. Dado que este estudio trata de elaborar un diagnóstico sobre la situación en la FAREM Chontales de Nicaragua, se siguió una distribución que representase, grosso modo, el panorama de la actividad académica del centro. El procedimiento para la selección de la muestra se hizo de forma representativa según el universo de estudiantes $(n=672)$ y profesores $(n=89)$ del que se partía para el estudio, 
distribuidas por carreras. Los Grupos de Discusión se compusieron de entre 8 y 10 participantes, hombres y mujeres, según la distribución por Carreras, turnos y Departamentos que se indica en la fig. 1. Todas las sesiones fueron grabadas, previo consentimiento informado de los participantes, para su posterior análisis.

En la literatura científica se considera inviable la posibilidad de utilizar esta metodología con menos de 4 participantes y más de 12. La propia metodología de los grupos de discusión obliga a planteamientos muy generales, esto es, a circunscribir el ámbito de la discusión pero dejando abierto su contenido. En los grupos de discusión, en realidad no existen preguntas, se trata de debatir ampliamente y sin tapujos. El coordinador de la actividad debe orientar y guiar pero no establecer preguntas directas, por eso se plantean cuestiones generales no cerradas. Es posible y probable que se obtengan resultados no previstos porque la riqueza de la investigación con este procedimiento es precisamente que es muy cualitativo y abierto. No obstante, el moderador ha de resituar constantemente las intervenciones para obtener información relevante para el estudio. En este sentido, diseñamos varios tópicos fundamentales sobre los que tratamos de potenciar la intervención de los participantes para conseguir información de nuestro interés para la investigación: Desconocimiento generalizado en la población estudiantil universitaria en zonas rurales y urbanas; Diferencias entre zonas e intergeneracionales; Ideas y mitos sobre rechazo social asociado a personas con VIH; Posible discriminación laboral a la que se ven sometidas las personas con VIH; Eficacia de las campañas informativas y, en su caso, cómo mejorarlas; El papel social y educativo de la Universidad; Información y carga influyente desde la familia, los medios de comunicación y los centros educativos; Uso y accesibilidad de métodos de barrera, como el preservativo; Lacra social y comunitaria: exploración sobre posibles actitudes de machismo enfocadas a los hábitos sexuales; Percepción sobre las posibles causas de transmisión del virus de inmunodeficiencia humana; Homosexualidad y rechazo. En la fig. 2 se refiere el sistema de codificación depurado y validado por los investigadores participantes de este estudio. Dicho sistema ha sido diseñado ad hoc para someter a análisis los debates realizados en los diferentes grupos de discusión.

\section{RESULTADOS}

A continuación presentamos los principales resultados del análisis cualitativo de los debates realizados en los grupos de discusión. Para ello se ha empleado la técnica de análisis de contenido y el programa de análisis cualitativo Atlas.ti, creando previamente el sistema de categorías y posteriormente los códigos de análisis. En el tratamiento informático del análisis, hemos obtenido como resultado una red conceptual de códigos relacionados.

\section{Desconocimiento generalizado entre la población estudiantil universitaria: zonas rurales vs zonas urbanas y diferencias intergeneracionales}

Respecto al conocimiento general analizado en las respuestas de los estudiantes, se ha observado que ellos mismos son conscientes de la falta de conocimiento que padece la sociedad en su conjunto y de cómo ello repercute considerablemente en el rechazo social hacia las personas con VIH: "A veces se dice... Si te sientas en el mismo asiento de la persona, te puedes contagiar. Hay muchas, muchas falsedades al respecto" (Grupo de alumnos 1, GA1). "Cuando alguien se 
da cuenta de que una persona está con esa enfermedad le empiezan como a rechazar, a restringir de la sociedad" (Grupo de alumnos 2, GA2). "Cuando miramos a alguien que tiene esa enfermedad creemos que es algo que sólo con que nos toque nos va a contagiar” (GA2).

Existe también la creencia de que los jóvenes están mejor informados que los adultos, aunque esa información no se correlaciona con la asunción de prácticas seguras, sino que más bien parece darse a entender que el mayor conocimiento no se traduce en cambios comportamentales: [los jóvenes están más informados] "porque vienen a la escuela, las Universidades, la tele..., pienso pues, que pueden estar más informados que los adultos" (GA2). "Pero se debe hacer acá una campaña fuerte para mejorar la visión y percepción sobre esta enfermedad" (Grupo de profesores, GP1). "Existe más información que antes, o sea el joven cuenta con más información, pero lamentablemente si usted levanta una encuesta los jóvenes... a pocos les gusta usar el condón. Es un problema cultural, diría yo. Le voy a poner un ejemplo, mi sobrino tiene dieciséis años y ya tiene dos niñas. Deduzca usted, y a pesar de reiterados consejos de que tomara medidas, nada, que no le gusta, dice que siente más deleite, más placer sin condón. Así de sencillo" (GP1).

Respecto a las diferencias entre el nivel de conocimientos de personas procedentes de zonas rurales o urbanas, se aprecia una mayoría de comentarios en el sentido de que en las zonas urbanas se da un mayor nivel de conocimientos que en el ámbito rural, donde el desconocimiento sobre los distintos aspectos de la pandemia es más acusado: "En el caso del casco rural surge desconocimiento sobre ese tema y es ahí donde se debería de profundizar bastante porque esas personas no adquieren muchas veces lo que aqui en las Universidades" (GA1). "Está el sector rural, que tienen desconocimiento de cómo es la transmisión, entonces ellos tienen más miedo del SIDA" (GA2). "En el caso rural, recuerden que tenemos una inmigración intensa de la parte rural a la parte urbana. Se han ido formando anillos de población campesina allí, donde prolifera posiblemente por el bajo nivel académico y la poca posibilidad laboral prolifera prostitución, prolifera droga, prolifera alcoholismo [...] Eso está en esos anillos de miseria, entonces además, por la ignorancia, hace que haya mayor impacto en esta población campesina que se ha venido desplazando a la parte urbana" (GP1).

\section{Ideas/mitos sobre rechazo social asociado a personas con $\mathrm{VIH}$}

La idea de rechazo social asociado a personas con VIH queda patente en las expresiones de los estudiantes y profesores al confirmar -desde sus experiencias y/o valores aprendidos- que existen en la actualidad conductas de rechazo y segregación hacia el colectivo enfermo con dicha patología. Hacen alusión a los mitos aun existentes y el desconocimiento de la enfermedad como posibles razones por las que dichas personas reciben un trato diferenciado por la sociedad en su conjunto: "Es obvio que la mayoría de las personas de Juigalpa tienen un tremendo rechazo a esas personas [...] Muchos tabús, muchos mitos que hay [...] entonces muchas de las pobres personas que tienen esa enfermedad son víctimas" (GA1). "Creo que, debido a la falta de educación, hacemos mal a estas personas y no les ayudamos" (GA2). "Es que las personas que tienen el SIDA son objeto de discriminación por la sociedad" (GP1). 


\section{Discriminación laboral / en el lugar de trabajo}

Profundizando en la misma cuestión, en lo que se refiere a la discriminación laboral o en los lugares de trabajo, los argumentos dados por los estudiantes son sencillos: cuando se conoce la existencia de alguna persona enferma con VIH, directamente la despiden o bien no es contratada. El resumen extraído del Verbatim pone de manifiesto que la población es consciente del desconocimiento de la enfermedad y que ello se transfiere a una falta de empatía hacia estas personas: "Si una persona en el trabajo se da cuenta de que alguien tiene el SIDA, el jefe inmediatamente lo despide" (GA1). "La gente de las instituciones debería estar más sensibilizada con esto porque si hay un trabajador de una institución privada o lo que sea, y se dan cuenta de que tiene esta enfermedad, a los dos días no tiene el empleo (GP1).

\section{Eficacia de las campañas informativas sobre el VIH y propuestas de mejora}

En el debate generado respecto a la eficacia de las campañas informativas y cómo poder mejorarlas, hemos observado mucha confianza en los mass media, como un buen vehículo para elevar el conocimiento de la población. Los participantes de los distintos grupos de discusión aprecian cualquier debate informativo, charla, curso etc., como forma propicia para conocer y aprender más sobre el virus y/o la enfermedad, aunque ponen de manifiesto que la información no es formativa per se, sino que es necesario una mayor profundización en el diseño de las campañas y actividades para que estas resulten motivadoras: "Hacerles llegar la información por medio de los canales televisivos. Con dramatización, por la radio. Porque hay radios que te permiten dar la charla por lo menos, porque no es lo mismo agarrar un folleto y leerlo que la dramatización. Y también lo de llegar, darle la información a la gente de qué es lo que necesitan para ir al examen, que no tengan miedo..." (GA2).

Aportan como dato destacable la necesidad de contar con la participación de personas con experiencias ligadas al desarrollo de la enfermedad para que puedan transmitir el mensaje a las generaciones jóvenes "de viva voz": "Las personas con ese tipo de enfermedad cuentan sus historias y son historias que llenan, porque uno los mira y ve que han sido golpeados en la vida. Por ejemplo, yo era una que no hacía caso ni decía nada. Pero en cambio cuando ese señor vino a mí clase, sí me llamó la atención. Entonces considero que debe ser una persona que esté con la enfermedad para que transmita todo el sufrimiento y todo lo que causa tener esa enfermedad" (GA2).

\section{El papel social y educativo de la Universidad}

Sobre la función social y educativa que la Universidad debe desempeñar, los participantes opinan que, aunque el conocimiento aprendido en dicho contexto es de valor para ellos mismos, la idea principal a desarrollar radica en la posibilidad de que los universitarios se conviertan en mediadores en salud. Ponen especial énfasis en la transferencia de los conocimientos aprendidos y desarrollados en la Universidad en materia de Educación para la Salud hacia el conjunto de la población para contribuir al desarrollo comunitario y a la no discriminación de personas enfermas con VIH: "Nosotros como jóvenes universitarios, que hemos tenido ya capacitación sobre eso, tenemos que hacernos comunicadores de las indicaciones para que la gente se sienta en sociedad, no sea que la sociedad más bien rechace a ese tipo de persona" (GA1). "Creo que lo 
mejor es capacitarse bien y a un determinado grupo para que luego la Universidad, a través de los estudiantes, pasase la información clara y necesaria para el resto de los habitantes" (GA2).

\section{Papel de la familia, los medios de comunicación y la Escuela}

Tanto estudiantes como profesores entienden que es en la familia donde se ponen en marcha los cimientos del aprendizaje en Educación Sexual. De una parte la familia, de otra la escuela y, junto a estos dos agentes socializadores, los medios de comunicación. Dichos ámbitos, como mecanismos de socialización, poseen un enorme potencial para generar conocimiento. Es por ello que estudiantes y profesores opinan que generalmente han sido sus propias familias quienes les han introducido en Educación Sexual, prevención, conocimiento de Infecciones de Transmisión Sexual (ITS), etc: "A mí la formación no me la ha dado ningún profesor ni ningún doctor. A mí me la ha dado mi padre. Yo pienso que eso es básico para erradicar los tabús y los miedos. Y me la dio mi padre cada paso y cada qué no debo hacer. Y se lo agradezco mucho porque he llevado una vida sana, gracias a Dios" (GA1). "mis papás me han enseñado y siempre con ese cuidado de decir si no te lo enseñamos nosotros, quién te las va a enseñar ¿no?”(GA2).

Además, consideran a la Escuela como una institución donde se introducen a los niños y niñas en Educación Sexual, para poder más adelante pasar a la vida sexual activa con un nivel mínimo de conocimiento: "Bueno, ese tema generalmente aquí lo recibimos desde los primeros años y después pasamos a la Secundaria y seguimos viendo el tema y llegamos a la Universidad inclusive" (GA2). "Normalmente si hay campañas en todas las escuelas de Secundaria y de Primaria” (GA2).

El carácter influyente que poseen los medios de comunicación lo convierten en una herramienta con un gran potencial divulgativo, aun cuando muchos opinan que esta divulgación solo se refiere a la publicación de datos estadísticos sobre afectados y no con otros objetivos más pedagógicos: "lo único que hacen es dar a conocer cantidad de gente que está infectada y no hacen pues... como apoyar a esa gente dando capacitación". "Se requiere un papel más activo de los medios de comunicación para concientizar a la población, para que sirvan como activo, para que la gente conozca el problema, porque esto es muy importante" (GA1).

\section{Uso y accesibilidad de métodos de barrera como el preservativo}

Aun cuando el método anticonceptivo de barrera más utilizado hoy día es el preservativo son los jóvenes los más reticentes a su uso, ya sea para evitar embarazos no deseados o para minimizar el riesgo de exposición a ITS. Existe una idea generalizada en las respuestas analizadas respecto al cambio de papeles que se ha dado sobre su uso. Hoy en día parece ser un método de barrera más usado por adultos que por jóvenes. El motivo acusado sobre dicho cambio de papeles parece justificado por la falta de conciencia y de conocimiento de los jóvenes: "Ahora los jóvenes son los más tercos. Tiempo atrás eran los adultos ahora no, ahora los jóvenes, que invirtieron un papel" (GA1). "Coincido con los compañeros en que la mayor incidencia se da en los jóvenes que inician a temprana edad su aventuras sexuales y, aunque ellos pudieran tomar precaución, no la toman porque no sienten el mismo placer" (GP1).

Respecto a la accesibilidad a los preservativos, el análisis revela que tanto jóvenes como adultos 
saben bien dónde y cómo conseguirlos de forma gratuita: "Eso lo regalan en el hospital. En los centros de salud, aqui los obsequian" (GA1).

\section{Posibles causas de la transmisión del Virus de Inmunodeficiencia Humana}

Las causas de transmisión del VIH parecen evolucionar a medida que cambian las prácticas imperantes en la sociedad. Al menos éste es el análisis que estudiantes y profesorado extraen al debatir sobre ello. Hay una gran parte que piensa que el medio más frecuente de transmisión de la enfermedad es mediante el uso compartido de jeringuillas a través de los tatuajes. Mientras tanto, la poca existencia de drogadictos que se administren sustancias por vía intravenosa en la zona, sigue relegando esta práctica a una forma de transmisión muy minoritaria, contrariamente a lo que ocurre en otros contextos sociales: "Los drogadictos aquí son contados, pues es que la droga es dificil de conseguir. Por aguja, no. Por aguja se puede dar bastante por los tatoos. Y como estamos en el mismo grupo..., venga pues, trae para acá, y entonces la misma aguja que utilizó él". "Hoy se dan mucho los tatuajes entre los jóvenes. Y claro, no se sabe higiénicamente si eso está controlado o no" (GP1).

\section{Actitudes de machismo enfocadas a los hábitos sexuales}

A grandes rasgos, el machismo es una de las lacras sociales más acusadas por los participantes del estudio. Si bien hay mucha población joven con niveles educativos secundarios $\mathrm{y}$ universitarios, sigue existiendo una forma machista de concepción de la sexualidad, de las prácticas de sexo y de los deberes de la mujer respecto a la protección. Para la mayor parte de los estudiantes y profesores, el hombre tiene mayores índices de prevalencia sobre la mujer en cuanto a decisiones sobre hábitos sexuales y medios de protección. Desde esta postura, la sociedad sigue estancada en una mayoría machista cuya idea principal es el placer en sus hábitos sexuales a costa de la sumisión de la mujer. Además, en ella se deposita toda la responsabilidad de las ITS o embarazos no deseados: "Bueno, pero el problema también es el machismo, Nicaragua es un país machista. Y ahi pues... que se protejan las mujeres, que se inyecten, que se tomen pastillas para no embarazarse", "Hay mucho machismo. Los hombres creen que su responsabilidad es cuidarnos y si salimos es nuestra culpa y no tiene que ser asi”" (GA1).

\section{Homosexualidad y rechazo}

En el análisis de las ideas más comunes entre jóvenes y adultos sobre la homosexualidad se pone de manifiesto que sigue siendo un tema tabú y que los homosexuales sufren una fuerte discriminación debido a su orientación sexual. De la misma forma, se sigue relacionando la idea de homosexualidad con la transmisión y contagio del VIH: "Bueno, en el caso de los homosexuales es un tema poco tocado ¿no? Pero cuando se da también es casi como hablar del SIDA, los van excluyendo porque los miran como personas raras [...] Pero en la sociedad en la que vivimos, la visión de ellos es tan negativa que también esas personas a veces tienden a vivir en el anonimato, su forma de ser la ocultan” (GA1).

Como avanzábamos al inicio de este apartado, los datos cualitativos de los grupos de discusión se seleccionaron y analizaron en Atlas.ti, según la relevancia del contenido. En la fig. 3 puede verse un ejemplo de cómo se ha desarrollado el estudio, párrafo por párrafo y código por código. En un 
análisis exhaustivo de los códigos utilizados, observamos que el desconocimiento básico que poseen los estudiantes y profesores es la etiqueta que aparece con mayor frecuencia ( $\mathrm{DESBA}=33)$, le siguen el conocimiento en educación formal $(\mathrm{CONEF}=19)$, discriminación social (DISOC $=16$ ), causas de transmisión $(\mathrm{CAUTRAN}=14)$, uso del preservativo (USPRE=10) y medios de influencia como la familia (MIFAM=10). El código relacionado con el papelresponsabilidad social y educativo de la Universidad (PRUSED) ha sido el de menos ocurrencia (fig. 4).

En la fig. 5 se muestra la red de códigos con mayor frecuencia y las relaciones que se establecen entre ellos. En todos los casos se aprecian asociaciones de códigos con entre cuatro, cinco y seis relaciones. El desconocimiento básico de la enfermedad queda relacionado con el conocimiento proveniente de la educación formal, con el uso del preservativo, con las causas de transmisión de Infecciones de transmisión sexual (ITS), con la discriminación sexual, los medios de influencia como los medios de comunicación y la discriminación social. Esta amalgama de conceptos y códigos forman un todo relacionado y complejo que describe crudamente la realidad a la que aun nos enfrentamos. Los datos muestran que el nivel de conocimiento sobre ITS sigue siendo bajo. En tanto que los medios de comunicación no desempeñan íntegramente su papel socializador, nos encontramos con una población carente de significados en Educación Sexual, donde aun se siguen dando conductas de rechazo hacia las personas enfermas. De otra parte, el código USPRE, relacionado con el uso del preservativo, ha sido el más relacionado en toda la red. Parece ser que dicho método de barrera marca el nivel de conocimiento de una persona respecto a la transmisión del VIH y de otras ITS, a la vez que se relaciona con las causas de transmisión, discriminación social y homosexual.

\section{DISCUSIÓN Y REFLEXIONES FINALES}

Los estudiantes -aun habiendo recibido educación básica sobre ITS y concretamente sobre VIHsiguen mostrándose más reacios que los adultos en cuanto al uso del preservativo. Aunque es el método de prevención más utilizado y hay que recordar que es posiblemente el mejor y más fiable producto de que disponemos hoy en día para protegernos de las ITS, sin embargo muchos jóvenes rechazan su uso. Estos resultados son congruentes con los datos aportados por numerosas investigaciones desarrolladas a lo largo de todo el mundo (Buhi y Goodson, 2007; Fergis, Zimmerman y Caldwell, 2007; Moreno, Rivera, Robles, Barroso, Frías y Rodríguez, 2008; Vinaccia, Quinceno, Gaviria, Soto, Gil y Ballester, 2007). Seguramente, en la base de esta situación está la persistencia de la negativa radical de algunas instituciones religiosas, así como diversas preconcepciones o prejuicios (incomodidad, alergias, pérdida de la sensibilidad) que condicionan el rechazo a su utilización (Terán-Ángel y Silva, 2012). Según los estudiantes, este hecho se relaciona también e intrínsecamente con las ideas machistas sobre su uso y el papel de la mujer, a la que se hace responsable única en el imaginario colectivo de la transmisión de las enfermedades venéreas. Tal vez esta situación haya generado la mayor percepción del riesgo que ha desarrollado la mujer frente al hombre que se ha detectado en otras investigaciones, aunque este último extremo no lo hemos podido corroborar en nuestro estudio.

Los conocimientos son considerados un factor de protección frente al VIH. Sin embargo, la investigación preexistente manifiesta constantemente la necesidad de que los adolescentes 
cuenten no solamente con información sobre el tema, sino también con las habilidades necesarias en materia de sexualidad, puesto que se ha demostrado que la información no garantiza, en sí misma, la adopción de medidas preventivas (Uribe, Valderrama, Sanabria, Orcasita y Vergara, 2009). El estudiantado y profesorado de Chontales admite, en este sentido, que las campañas informativas y cursos de capacitación son efectivos en cuanto a nivel personal pero que, a posteriori, son pocas las personas que ponen en práctica lo aprendido, siendo menor la incidencia en las generaciones más jóvenes y en las de menor formación. La importancia del medio comunicativo y tecnológico a través del cual se enseñe a la población es un tema interesante para jóvenes y adultos, y que se refiere reiteradamente en las discusiones. En este sentido, ambos grupos poblacionales dicen preferir los distintos medios de comunicación como radio y televisión para la emisión de programas educativos de Educación para la Salud y Educación Sexual por su impacto, influencia y velocidad de transmisión. Igualmente se ha puesto de manifiesto la importancia de la familia como institución socializadora, encargada de manera más o menos generalizada de generar la comunicación con los hijos e hijas en el fomento de aprendizajes referidos a nociones básicas de educación sexual e ITS. Se sigue considerando, en este sentido, a la familia, la Escuela y al propio grupo de iguales, como los principales responsables de la transmisión de los elementos culturales fundamentales y más básicos en esta materia.

Uno de los principales problemas percibidos por los participantes del estudio, a nivel general, es la persistencia de numerosos mitos con relación a las personas portadoras de VIH o que directamente han desarrollado la enfermedad. Se siguen perpetuando mitos sobre las personas y también sobre las formas de contagio existiendo, en este sentido, una alta correlación percibida con el nivel de conocimiento y formación que posee la persona. Ello provoca que sea la discriminación social un tema de interés comunitario. Los estereotipos asociados tradicionalmente al VIH continúan jugando un importante papel en la justificación, desarrollo y mantenimiento de la estigmatización hacia las personas seropositivas (Brooks, Martín, Ortiz y Veniegas, 2004; Dijker, Kok y Koomen, 1996). Esta estigmatización se da tanto en lo físico y social como en lo verbal e institucional, siendo especialmente dramática esta última, lo que conlleva dificultades de acceso a la vivienda, la educación o el mundo del trabajo. En concreto, los aspectos laborales son expresamente referidos en los grupos de discusión, habiendo un alto consenso en el sentido de que ser seropositivo es directamente incompatible con estar trabajando en este contexto.

Uno de los factores que han sido destacados con relación al estigma es la homosexualidad. Los participantes estiman que la homosexualidad es ya, en sí misma, un motivo de discriminación por parte de la sociedad hacia este colectivo por lo que, si lo unimos a VIH, e ITS, los tabús, mitos y la discriminación se agravan aun más. Esta percepción de la población juigalpina nos parece que excede totalmente del marco estudiado, siendo extrapolable a buena parte de la población mundial. Conviene recordar, en este sentido, las palabras de Sidibé:

"La falta de respuestas eficaces ha permitido que el VIH alcance niveles de crisis en muchas comunidades de hombres que tienen relaciones sexuales con hombres y transexuales. Los esfuerzos para revertir esta crisis deben ser informados con evidencia, fundados en los derechos humanos y sustentados por la despenalización de la homosexualidad" (Sidibé, 2009). 
Este trabajo, como todo el conjunto de actuaciones que venimos desarrollando en la FAREMChontales de Nicaragua, se posiciona en la defensa de la efectividad de los diálogos transdisciplinarios en el sentido que defienden Filho et al (2009), esto es, en la capacidad de identificar problemas prácticos que agreguen el mayor número posible de intereses sociales y de organizar en torno a esos problemas los esfuerzos de intervención (Filho, Castiel y Ayres, 2009). En concreto, la detección de varias evidencias: la necesidad de seguir interviniendo formativamente en materia de Educación para la Salud mediante acciones mucho más formativas que informativas, el papel que los medios de comunicación pueden llegar a jugar en este contexto, el importante rol que la familia juega en materia de información (que sugiere la necesidad de hacer también promoción de la salud, por y para las propias familias), el reto pendiente de la homosexualidad como tabú y lacra social, especialmente en el ámbito laboral son algunas de las líneas directrices que muestran el camino a seguir en los próximos años.

La estructura académico-investigadora que, ex profeso, la UNAN-Managua decidió constituir para abordar y desarrollar en profundidad todas las temáticas relacionadas con la Salud, el Desarrollo y la Calidad de Vida, bebe de estos planteamientos y especialmente de la idea de que la construcción de un espacio de trabajo y educación saludable es la mejor fórmula para que esas experiencias sean trasvasadas posteriormente a la vida laboral y familiar de los futuros profesionales. Además, no hay que olvidar el papel de cambio y transformación que los universitarios pueden desarrollar en su rol de agentes mediadores, como el propio estudiantado ha señalado en este trabajo. Nos gustaría destacar también el valor de la percepción del riesgo como elemento a partir del cual construir el edificio preventivo, por cuanto que el riesgo no depende sólo del cálculo de probabilidades sino también, y muy especialmente, de los contextos sociales y culturales (Torres-Nerio, Domínguez-Cortinas, Van't Hooft, Díaz Barriga y Cubillas-Tejeda, 2010).

En la idea de seguir desarrollando acciones de investigación como la que ahora presentamos, que vayan mucho más allá de la especulación teórica y que realmente sirvan a la intervención social desde una perspectiva eminentemente participativa, que supongan orientaciones que conduzcan a actuaciones de intervención realistas y que, a la postre, impulsen un impacto en la mejora de la salud y calidad de vida de toda la comunidad, trataremos de seguir trabajando conjuntamente en los próximos años. 


\section{BIBLIOGRAFÍA}

Brooks, R.A.; Martín, D.J.; Ortiz, D.J. Y Veniegas, R.C. (2004). Perceived barriers to employment among persons living with HIV/AIDS. Aids Care. 16:756-766.

Buhi, E.R. Y Goodson, P. (2007). Predictors of adolescence sexual behavior and intention: A theory-guided systematic review. Journal of Adolescence Health. 40: 4-21.

Dijker, A.J.; Kok, G. Y Koomen, W. (1996). Emotional reactions to people with AIDS. Journal of Applied Social Psychology. 26:731-748.

Fergus, S., Zimmerman, M. A. Y Caldwell, C.H. (2007). Growth trajectories of sexual risk behavior in adolescence and young adulthood. American Journal of Public Health. 97:1096-1101.

Filho, N.A.; Castiel, L.D. Y Ayres; J.R. (2009). Riesgo: concepto básico de la epidemiología. Salud Colectiva. 5(3):323-344.

Florez, M. Y Mendoza, R. (2001). El SIDA: su percepción de riesgo de contagio en estudiantes universitarios. Educación y Ciencia, Revista de la Faculta de Educación de la Universidad Autónoma de Yucatán. 5 (23):53-66.

Gobierno de Nicaragua (2010). Informe nacional sobre los progresos realizados en la aplicación del UNGASS, Nicaragua 2008-2009. Managua: Comisión nicaragüense del SIDA.

López, F.; Cobos, D. Y Morón, J. A. (2011). Un proyecto de investigación e intervención socioeducativa: Observatorio de Calidad de Vida, Desarrollo y Educación para la Salud de la UNAN-Managua. II Jornada Monográfica de la Sociedad Iberoamericana de Pedagogía Social. Madrid (España), 29 de abril.

May, R. M. Y Anderson, R. M. (1979). Dynamic biology of infectious diseases. Nature. 280:455-461.

Moreno, D.; Rivera, B.; Robles, S.; Barroso, R.; Frías, B. Y Rodríguez, M. (2008). Características del debut sexual de los adolescentes y determinantes del uso inconsistente del condón desde el análisis contingencial. Psicología y Salud.18: 207-225.

Morón, J. A.; López, F. Y Cobos, D. (2011). La problemática del SIDA. Una aproximación socioeducativa. Sevilla: Universidad Nacional Autónoma de Nicaragua y Universidad Pablo de Olavide.

Morón, J. A.; López, F. Y Cobos, D. (2011). El Observatorio de Calidad de Vida, Desarrollo y Educación para la Salud de la UNAN-Managua. $V$ Congreso de Universidad y Cooperación al Desarrollo. Cádiz (España), 6-8 de abril. 
Noboa, P. Y Serrano-García, I. (2006). Autoeficacia en la negociación sexual: retos para la prevención de VIH/Sida en mujeres puertorriqueñas. Revista Latinoamericana de Psicología. 38:21-43.

Rodríguez, E.; Ballesteros, J.C.; Mejías, I. Y Rodríguez, M.A. (2008). La lectura juvenil de los riesgos de las drogas: del estereotipo a la complejidad. Madrid: Fundación de Ayuda contra la Drogadicción.

Terán-Ángel, G. Y Silva, N. (2012). Dificultades en la enseñanza de prácticas de sexo seguro y en la adherencia al uso del preservativo. Avances en Biomedicina. 1(1): 2-3.

Teva, I.; Bermúdez, Ma . P. Y Buela, G. (2009). Variables sociodemográficas y conductas de riesgo en la infección por el VIH y las enfermedades de transmisión sexual en adolescentes. Revista Española de Salud Pública. 83(2):309-320.

Torres-Nerio, R.; Domínguez-Cortinas, G.; Van'T Hooft, A.; Díaz Barriga, F.; Cubillas-Tejeda, A.C. (2010). Análisis de la percepción de la exposición a riesgos ambientales para la salud, en dos poblaciones infantiles, mediante la elaboración de dibujos. Salud Colectiva. 6(1):65-81.

UNAIDS (2010). Global report: UNAIDS Report on the Global AIDS Epidemic 2010. Geneva: Joint United Nations Programme on HIV/AIDS.

Uribe, A.; Valderrama, L.; Sanabria, A. Orcasita, L. Y Vergara, T. (2009). Descripción de los conocimientos, actitudes, susceptibilidad y autoeficacia frente al VIH/SIDA en un grupo de adolescentes colombianos. Pensamiento Psicológico. 12:29-44.

Uribe, A; Vergara, T. Y Barona, C. (2009). Susceptibilidad y autoeficacia frente al VIH/Sida en adolescentes de Cali-Colombia. Revista Latinoamericana de Ciencias Sociales, Niñez y Juventud. 7(2):1513-1533.

Vinaccia, S.; Quinceno, J.M.; Gaviria, A.M.; Soto, A.M.; Gil, M.D. Y Ballester, R. (2007). Conductas sexuales de riesgo para la infección por VIH/SIDA en adolescentes colombianos. Terapia Psicológica. 25:39-50.

WHO (2010). Towards universal access: scaling up priority HIV/AIDS interventions in the health sector: progress report 2009. Geneva: World Health Organization.

Declaración por Sr. Michel Sidibé, Director ejecutivo de ONUSIDA, en el Día Internacional contra la Homofobia, 17 de mayo. Sitio web de ONUSIDA. http://www.unaids.org/en/KnowledgeCentre/Resources/PressCentre/PressReleases/2009/2 0090515 


\section{AGRADECIMIENTOS}

En primer lugar a la Agencia Española de Cooperación Internacional para el Desarrollo (AECID) por haber financiado algunos de los trabajos preparatorios y la puesta en valor de los resultados que se recogen en este artículo, mediante el proyecto A1/040008/11, correspondiente al Programa de Cooperación Interuniversitaria e Investigación Científica (PCI), aprobado según Resolución de 16 de noviembre de 2011 (BOE 12/12/11). Muy especialmente, por su implicación personal, respaldo y constante apoyo, al equipo decanal, claustro de profesores y estudiantes de la FAREM-Chontales de la UNAN-Managua de Nicaragua, auténticos protagonistas de este trabajo. 


\section{Figura 1: Distribución de estudiantes y profesorado participante en los Grupos de Discusión}

\begin{tabular}{|c|c|c|c|c|}
\hline & & & & \\
\hline Carrera & Año & Turno & Sexo & Edad \\
\hline Ingeniería Agroindustrial & $2^{\circ}$ & Vespertino & Femenino & 18 \\
\hline Ciencias Ambientales & $2^{\circ}$ & Sabatino & Masculino & 21 \\
\hline Mercadotecnia & $1^{\circ}$ & Vespertino & Femenino & 21 \\
\hline Bioanálisis clínico & $2^{\circ}$ & Matutino & Femenino & 19 \\
\hline Administración de empresas & $2^{\circ}$ & Sabatino & Masculino & 22 \\
\hline Banca y Finanzas & $2^{\circ}$ & Nocturno & Masculino & 22 \\
\hline Biología & $2^{\circ}$ & Sabatino & Masculino & 25 \\
\hline Ingeniería Agroindustrial & $2^{\circ}$ & Vespertino & Femenino & 18 \\
\hline Ing. Industrial y de Sistemas & $2^{\circ}$ & Matutino & Masculino & 18 \\
\hline
\end{tabular}

Segundo Grupo de Discusión de alumnos (GA2) (n=10)

\begin{tabular}{l|c|c|c|c}
\hline \multicolumn{1}{c|}{ Carrera } & Año & Turno & Sexo & Edad \\
\hline \hline Informática educativa & $1^{\mathbf{0}}$ & Sabatino & Masculino & 19 \\
\hline Informática educativa & $1^{\mathbf{0}}$ & Sabatino & Femenino & 18 \\
\hline Bioanálisis clínico & $2^{\mathbf{0}}$ & Matutino & Femenino & 16 \\
\hline Contaduría pública y finanzas & $5^{\circ}$ & Nocturno & Femenino & 20 \\
\hline Contaduría pública y finanzas & $5^{\circ}$ & Nocturno & Femenino & 22 \\
\hline Educación física & $3^{0}$ & Matutino & Masculino & 28 \\
\hline Educación Física & $3^{\circ}$ & Matutino & Masculino & 30 \\
\hline Administración de empresas & $5^{\circ}$ & Sabatino & Masculino & 26 \\
\hline Administración de empresas & $5^{\circ}$ & Sabatino & Masculino & 20 \\
\hline Educación Física & $3^{\circ}$ & Matutino & Masculino & 25 \\
\hline \hline
\end{tabular}

\section{Grupo de Discusión de profesores (GP1) $(n=8)$}

\begin{tabular}{l|c|c|c}
\hline Departamento docente & Área & Sexo & Edad \\
\hline CC de la Educación y Human. & Psicología & Femenino & 60 \\
\hline Tecnología y Salud & Computación & Masculino & 38 \\
\hline Tecnología y Salud & Agroindustria & Femenino & 27 \\
\hline Tecnología y Salud & Química y Farmacia & Femenino & 33 \\
\hline CC de la Educación y Human. & Informática educ. & Masculino & 59 \\
\hline CC Económicas y Admtvas. & Ciencias Económicas & Masculino & 37 \\
\hline CC Económicas y Admtvas. & Ciencias Económicas & Masculino & 41 \\
\hline CC Económicas y Admtvas. & Física-Matemáticas & Masculino & 36 \\
\hline \hline
\end{tabular}

Fuente: (Elaboración propia) 
Figura 2: Sistema de categorías y códigos de análisis de contenido empleados en la investigación

\begin{tabular}{|c|c|c|c|c|}
\hline $\begin{array}{c}\text { CATEGORÍ } \\
\mathbf{A} \\
\end{array}$ & DESCRIPCIÓN & $\begin{array}{c}\text { CÓDIG } \\
0 \\
\end{array}$ & $\begin{array}{c}\text { SUBCÓDIG } \\
\text { O } \\
\end{array}$ & $\begin{array}{l}\text { CÓDIGO } \\
\text { FINAL } \\
\end{array}$ \\
\hline 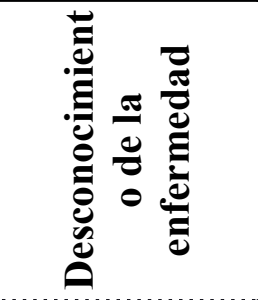 & $\begin{array}{l}\text { Desconocimiento generalizado } \\
\text { de conceptos básicos sobre } \\
\text { VIH }\end{array}$ & DES & BA & $D E S B A$ \\
\hline \multirow{3}{*}{ 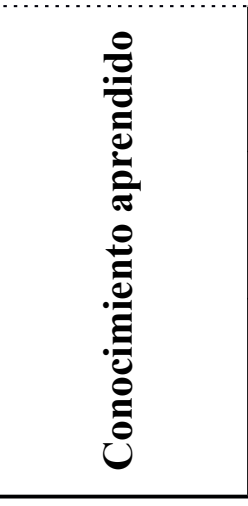 } & $\begin{array}{l}\text { Conocimiento proveniente de } \\
\text { educación } \\
\text { (Universidad) }\end{array}$ & $\mathrm{CON}$ & EF & CONEF \\
\hline & $\begin{array}{l}\text { Conocimiento proveniente de } \\
\text { educación no formal (familia y } \\
\text { grupo de iguales) }\end{array}$ & $\mathrm{CON}$ & NF & CONF \\
\hline & $\begin{array}{l}\text { Conocimiento proveniente de } \\
\text { los medios de comunicación de } \\
\text { masas (TV) y redes sociales } \\
\text { (Facebook, Twitter, y otros) }\end{array}$ & $\mathrm{CON}$ & MC & CONMC \\
\hline :อ气 & Discriminación social & DI & \multirow[t]{2}{*}{ SOC } & DISOC \\
\hline 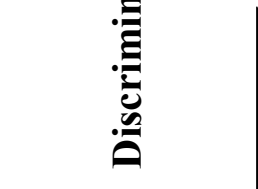 & Discriminación laboral & DILAB & & $D I L A B$ \\
\hline \multirow{2}{*}{ U.: } & $\begin{array}{l}\text { Eficacia de campañas } \\
\text { informativas }\end{array}$ & ECAM & IN & ECAMIN \\
\hline & $\begin{array}{l}\text { Mejora de las campañas } \\
\text { informativas }\end{array}$ & MCAM & IN & MCAMIN \\
\hline ฮ. & $\begin{array}{l}\text { Papel-Responsabilidad } \\
\text { Social/educativa de la } \\
\text { Universidad }\end{array}$ & PRU & SED & PRUSED \\
\hline \multirow{2}{*}{ 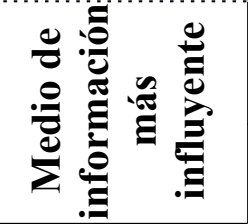 } & $\begin{array}{l}\text { Influencia procedente de la } \\
\text { familia }\end{array}$ & MI & FAM & $M I F A M$ \\
\hline & $\begin{array}{l}\text { Influencia procedente de la } \\
\text { escuela o grupo de iguales }\end{array}$ & MI & ESC & MIESC \\
\hline
\end{tabular}




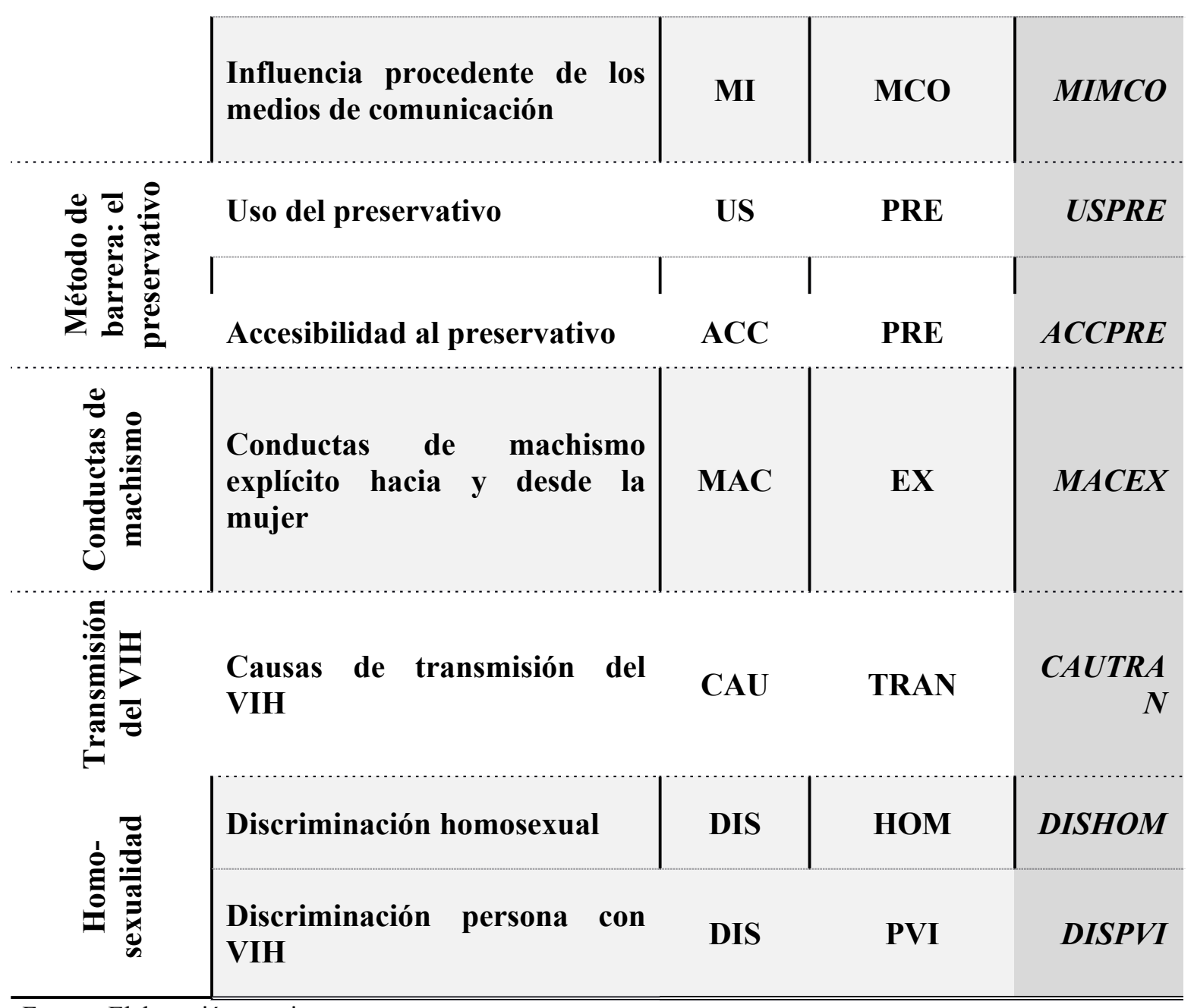

Fuente: Elaboración propia 


\section{Figura 3: Tratamiento informático de los contenidos con Atlas.ti. Un ejemplo sobre el} análisis de los datos desarrollado

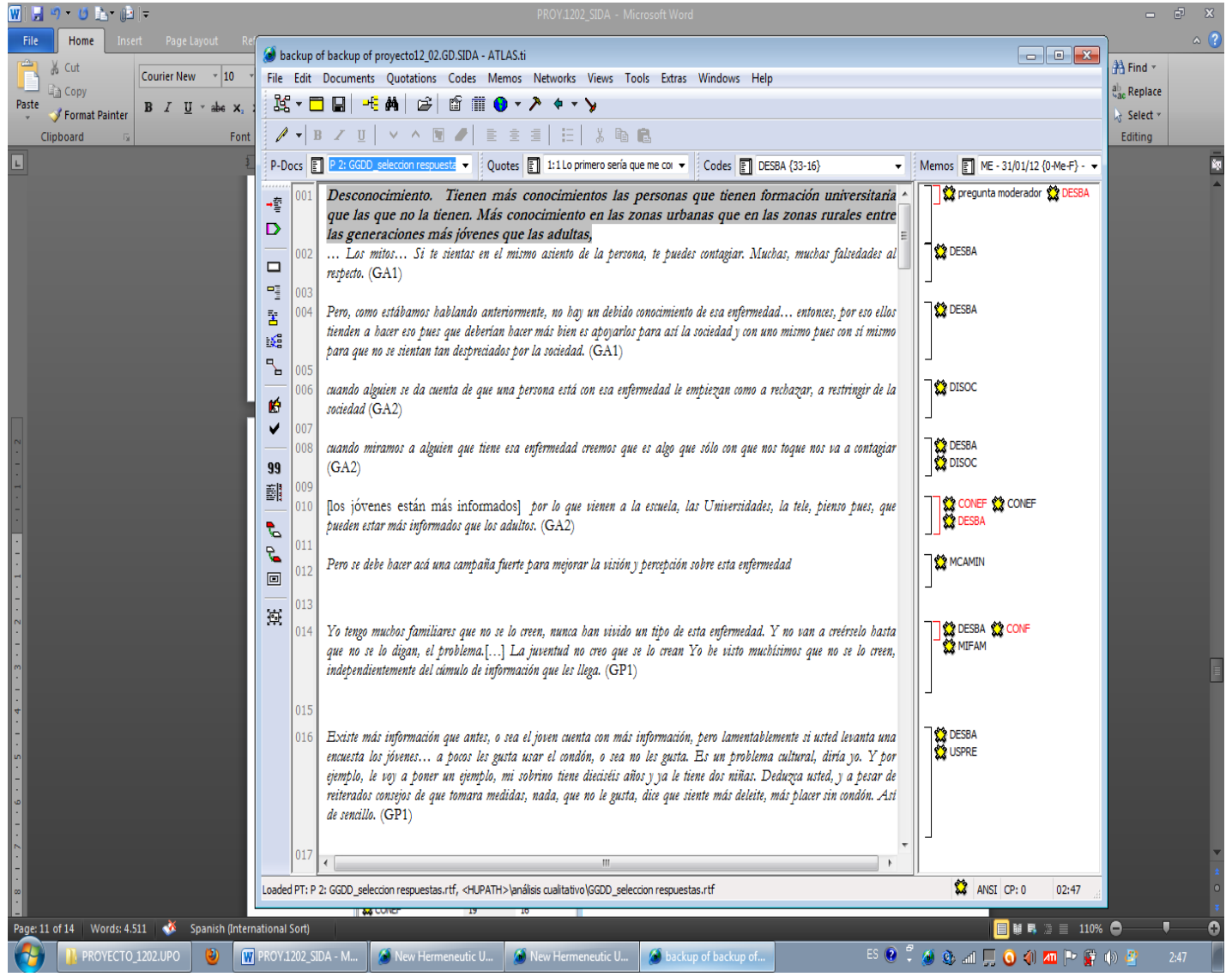

Fuente: Elaboración propia 


\section{Figura 4: Recuento de códigos utilizados y frecuencias de aparición}

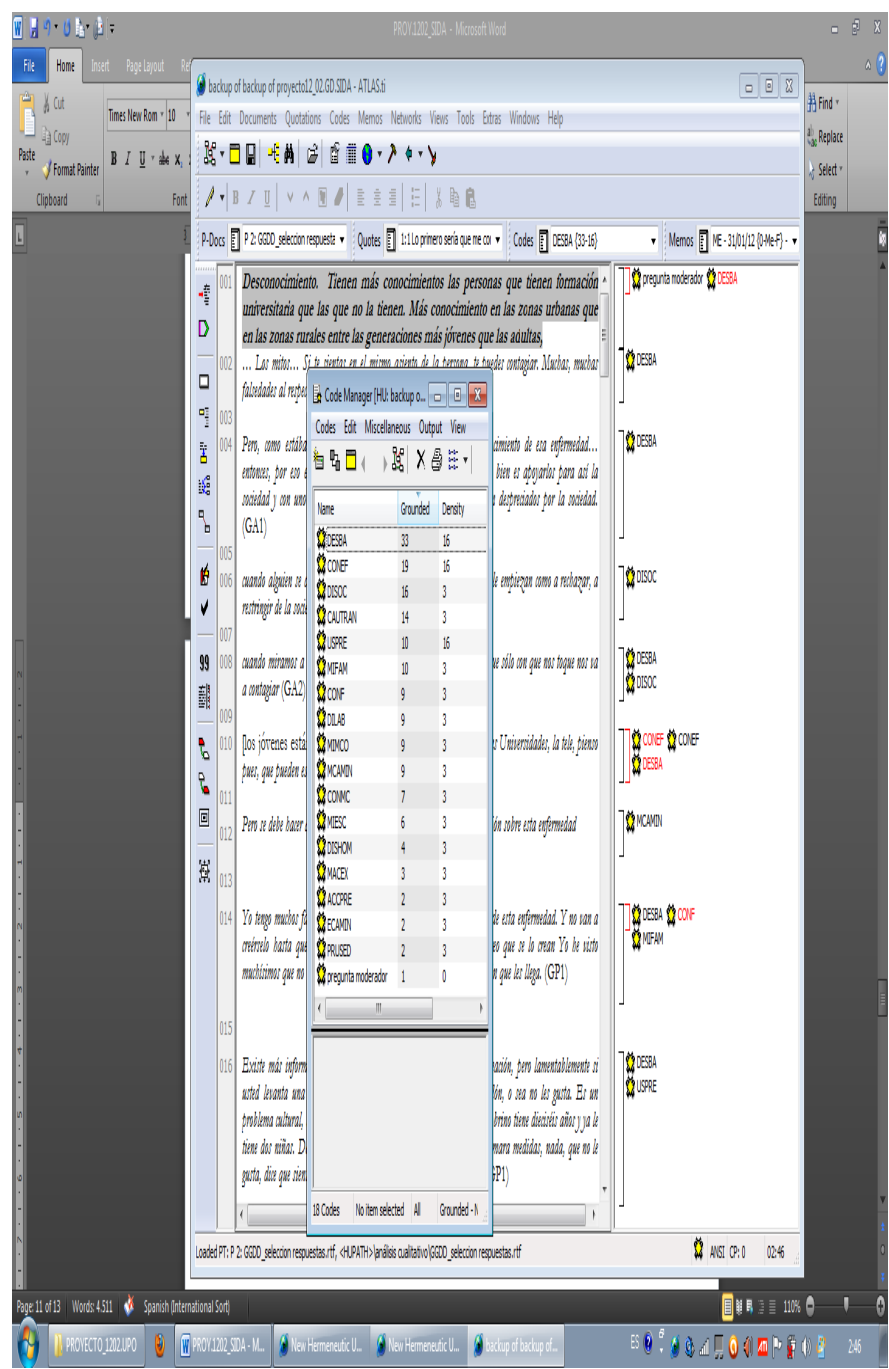

Fuente: Elaboración propia 
Figura 5: Red conceptual de códigos con mayor frecuencia de aparición en nuestra investigación e interrelaciones que se establecen

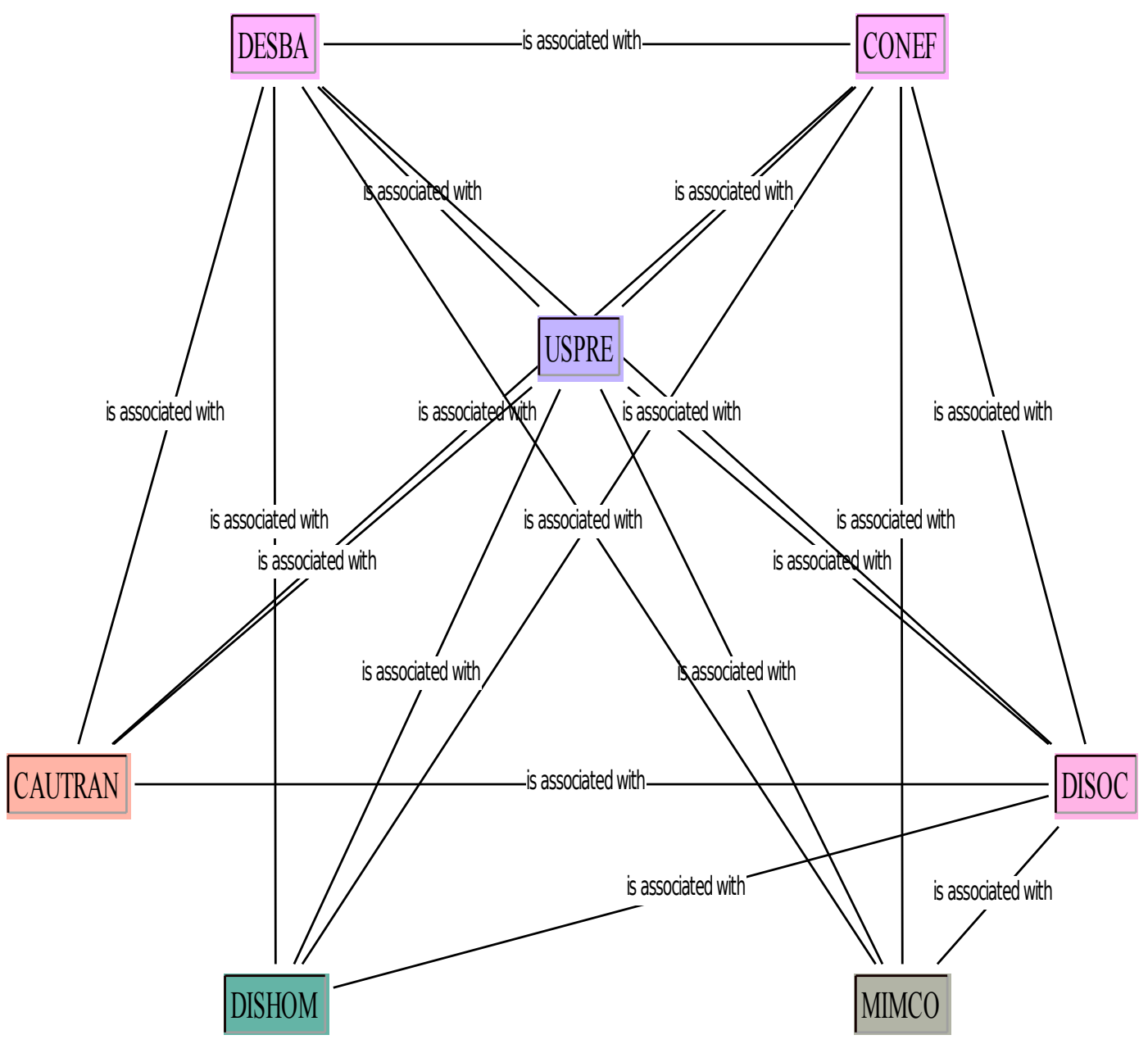

Fuente: Elaboración propia 